\section{UPPER GASTROINTESTINAL MALIGNANCIES: 6976 ENDOSCOPY REVIEW IN A MULTINATIONAL STUDY}

S K Roy Queen Mary's Hospital, Sidcup, Kent, UK

\subsection{6/gutjnl-2013-305143.27}

Introduction This is a multinational study to compare the prevalence of upper gastrointestinal malignant and premalignant conditions in three different populations. The populations involved in this study included British, Japanese and Arabian. It is clearly known that there are distinct variations in the lifestyle and habits of these different groups. Variables include their contrasting diet, smoking tendencies, alcohol intake and genetic pool. This study analyses figures from these different communities highlighting the prevalence of these very important upper gastrointestinal complaints. It is interesting to scrutinize these findings alongside the lifestyle and demographic traits of each society. This study endeavours to demonstrate the significant influence these demographic factors impose upon both malignant and premalignant upper gastrointestinal disease. Aims/Background To compare the prevalence of upper gastrointestinal (UGI) malignant and premalignant conditions in three separate populations; British, Japanese and Arabian.

Method 6976 Upper Gastrointestinal (UGI) endoscopies were retrospectively reviewed in a multinational comparative study. This involved three population groups: Group A- British \& others $(n=2158)$; Group B- Japanese $(n=2628)$; Group C- Arabians (Saudi Arabians \& others $(n=2190))$. The majority of the patients fell in the above 16 age group. The patients presented with UGI symptoms and were selected at random. The data was collected between 1986 and 2012.

The study involved patients from Barnsley District General Hospital (Barnsley), Darent Valley Hospital (Dartford) \& Queen Mary's Hospital (Sidcup) in UK-Group A; Showa University Fujigaoka Hospital \& Niigata Cancer Centre Hospital (Japan)Group B; Jubail Hospitals (Saudi Arabia) - Group C.

Results A comparison was made for the malignant and premalignant diseases. It revealed a very high incidence of UGI malignancies amongst Japanese (Group B). There is significant number of malignant diseases amongst non-Arabians, but such malignancies amongst Arabians are rare.

The incidence of gastric ulcers and gastric polyps are very high amongst Japanese compared to the other population groups in the study.

It is found that the prevalence of Barrett's Oesophagus has increased significantly in the last ten years.

The results are summarised as below:

UGI malignancies recorded:

Group A: British population: 37 (1.71\% with 95\% CI: 1.17 to 2.26)

Group B: Japanese population: 148 (5.63\% with 95\% CI: 4.75 to 6.51 )

Group C: Arabian population: 16 (1.17\% with 95\% CI: 1.17 to 2.26 )

Ulcers in the upper GI tract recorded:

Group A: British population: 366 (16.96\%, 95\% CI: 15.38 to 18.54$)$

Group B: Japanese population: 498 (18.95\% 95\% CI: 17.45 to 20.45)

Group C: Saudi population: $506(23.11 \%, 95 \% \mathrm{CI}=21.34$ to 24.87$)$.

Conclusion It can be concluded that Arabians (Saudis) suffer rarely from UGI malignancies and it may be related to social, environmental, geographical, genetic and dietary habits. Dietary habits in Japan are very different from the British and Saudi population.

Alcohol consumption amongst Saudis is very little. In comparison, Japanese and UK populations drink alcohol significantly more.

Prevalence of Barrett's has increased significantly in the last 10 years. It is likely due to effective Helicobacter Pylori eradication and due to increasing awareness of Barrett's amongst endoscopists. 